\title{
Antihyperon-Hyperon production in antiproton-proton annihila- tions with PANDA at FAIR
}

\author{
Michael Papenbrock ${ }^{1, a}$ \\ on behalf of the PANDA collaboration
}

${ }^{1}$ Dept. of Physics and Astronomy, Uppsala University, Box 516, S-751 20 Uppsala, Sweden

\begin{abstract}
Hyperon production is an excellent probe of QCD in the confinement domain, and spin observables are a powerful tool in understanding the underlying physics. For the $\Omega$ hyperon, seven polarisation parameters can be extracted from the angular distributions of its decay products with the future PANDA experiment at FAIR. Simulation studies reveal great prospects for strange and single charmed hyperon channels with PANDA. Software tools supporting these investigations are currently under development.
\end{abstract}

Hyperon production in $\bar{p} p \rightarrow \bar{Y} Y$ reactions gives important insights into strangeness and charm production. In particular, the production of single-, multi-strange and single-charmed hyperons from the light quarks in $\bar{p} p$ implies processes where they are replaced by heavier quarks. For any given process the relevant degrees of freedom are given by its energy scale, which for strangeness production is determined by the mass of the strange quark, $m_{s} \approx 100 \mathrm{MeV}$. This is close to the QCD cut-off, $\Lambda_{Q C D} \approx 200 \mathrm{MeV}$ [1], where the coupling constant $\alpha_{s}$ grows too large for perturbative QCD to be applicable. Therefore, the production of strange hyperons presents an excellent tool to study QCD in the region below the perturbative regime, i.e. the confinement domain, about which very little is known so far. On the other hand, the scale of charm production is governed by the mass of the charm quark, $m_{c} \approx 1300 \mathrm{MeV}$, which is more than ten times larger than $m_{s}$. In this region, the strong coupling constant becomes $\alpha \approx 0.3$, just barely enough for a perturbative treatment to be valid. Comparing the production of strange and charmed hyperons could give important insights into the differences in the production mechanism at these two energy scales. The production of hyperons in $\bar{p} p \rightarrow \bar{Y} Y$ is often described by theoretical models based on a constituent quark-gluon picture [2]. In case of strange hyperons, models based on the exchange of one or more $t$-channel kaons exist as well [3]. There have also been efforts to combine both approaches [4]. In order to discriminate between different models, spin observables are very powerful. For hyperons these can be related to the spin of the individual quarks. The $\Lambda$ hyperon is often modelled as consisting of a spin $0 u d$ di-quark and an $s$-quark carrying the hyperon spin. Since the $\Lambda_{c}^{+}$hyperon can be described in a similar way, comparing the spin observables of $\Lambda$ and $\Lambda_{c}^{+}$could give deeper insight into the role of spin degrees of freedom in the production of $s$ - and $c$-quarks.

The density matrix of a particle of arbitrary spin $j$ is given by

$$
\rho=\frac{1}{2 j+1} I+\sum_{L=1}^{2 j} \frac{2 j}{2 j+1} \sum_{M=-L}^{L} Q_{M}^{L} r_{M}^{L},
$$

a e-mail: michael.papenbrock@physics.uu.se 


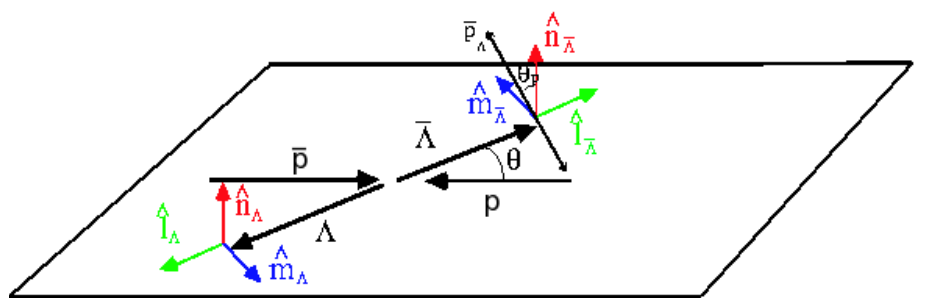

(a)

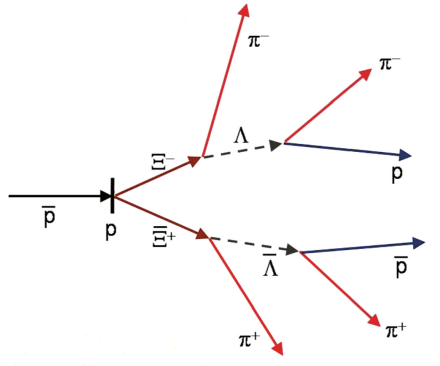

(b)

Figure 1. (a) The reference system of $\bar{p} p \rightarrow \bar{Y} Y$. (b) The $\bar{p} p \rightarrow \bar{\Xi} \Xi, \Xi \rightarrow \Lambda \pi, \Lambda \rightarrow p \pi$ decay.

in terms of the hermitian matrices $Q_{M}^{L}$ and the polarisation parameters $r_{M}^{L}$ [5]. Here, the first term represents the unpolarised differential cross section $I$ and the second the polarised part with the $r_{M}^{L}$ parameters, $L$ being the angular momentum and $M$ its third component. For spin $\frac{1}{2}$ particles the $Q_{M}^{L}$ are the Pauli matrices and the polarisation parameters correspond to the vector polarisations $P_{l}, P_{m}$ and $P_{n}$. The indices are defined in the coordinate system shown in the left panel of figure 1 . In parity conserving processes, e.g. $\bar{p} p \rightarrow \bar{\Lambda} \Lambda$ with unpolarised beam and target, $P_{l}$ and $P_{m}$ equal zero due to symmetries of the spin density matrix. The only non-zero polarisation component is the one perpendicular to the production plane, namely $P_{n}$.

Since the hyperons have a weak, parity violating decay, the decay products are preferentially emitted along the direction of the spin of the parent hadron. For $\Lambda \rightarrow p \pi^{-}$, the polarisation is accessible by measuring the angular distribution of the proton, both of which are related by $I\left(\cos \theta_{p}\right)=\frac{1}{4 \pi}\left(1+\alpha_{\Lambda} P_{n} \cos \theta_{p}\right)$, with $\alpha_{\Lambda}=0.64$ [6] being the asymmetry parameter. Some hyperons decay into other weakly decaying hyperons, e.g. $\Xi^{-} \rightarrow \Lambda \pi^{-}$(see right panel of figure 1), in which case additional asymmetry parameters become accessible through the angular distribution of the protons [7].

The spin structure of spin $\frac{3}{2}$ hyperons is more complicated. Fifteen $Q_{M}^{L}$ matrices with corresponding $r_{M}^{L}$ parameters become relevant. Eight polarisation parameters were found to be zero using symmetries imposed by the strong interaction [8]. In the $\Omega^{-} \rightarrow \Lambda K^{-}$decay the parameters $r_{0}^{2}, r_{1}^{2}$, and $r_{2}^{2}$ can be obtained from the angular distribution of the $\Lambda$ [8], assuming that $\alpha_{\Omega}=0$ according to previous measurements [6]. The combined angular distribution $I\left(\theta_{\Lambda}, \phi_{\Lambda}, \theta_{p}, \phi_{p}\right)$ of the $\Lambda$ hyperons from $\Omega^{-}$decay and the protons from the subsequent $\Lambda$ decay grants access to the other four non-zero polarisation parameters $r_{-1}^{1}, r_{-1}^{3}, r_{-2}^{3}$ and $r_{-3}^{3}$.

The major part of the existing data on $\bar{p} p \rightarrow \bar{Y} Y$ are collected with beam momenta below 2 $\mathrm{GeV} / c$. In this region, the PS185 experiment provided a high-quality data set for $\Lambda$ and $\Sigma$ hyperons [9]. The spin structure of the $\bar{p} p \rightarrow \bar{\Lambda} \Lambda$ reaction was explored in detail, but neither quark-gluon models nor hadron models were in agreement with the data on the spin observables [10]. For multistrange and charmed hyperons produced in $\bar{p} p$ annihilation the existing data are much more limited, with only a few bubble chamber events on $\bar{p} p \rightarrow \bar{\Xi}^{+} \Xi^{-}$and no data on $\bar{p} p \rightarrow \bar{\Omega}^{+} \Omega^{-}$nor $\bar{\Lambda}_{c}^{-} \Lambda_{c}^{+}$. New experimental data will serve as a guideline for the theoretical investigation which in turn could widen our understanding of the underling processes in this energy regime.

The foreseen PANDA experiment at FAIR provides unique possibilities for the investigation of hyperon physics. The HESR storage ring can be operated with antiprotons in a momentum range 
Table 1. Estimated production rates of various hyperon channels $[8,13]$. The cross sections marked with a $*$ have a very large uncertainty. Details are given in the text.

\begin{tabular}{llllll}
\hline Beam mom. $(\mathrm{GeV} / c)$ & Reaction & $\sigma(\mu \mathrm{b})$ & Eff $(\%)$ & Decay & Rate at $1 \cdot 10^{31} \mathrm{~cm}^{-2} \mathrm{~s}^{-1}$ \\
\hline 1.64 & $\bar{p} p \rightarrow \bar{\Lambda} \Lambda$ & 64 & 11 & $\Lambda \rightarrow p \pi^{-}$ & $29 \mathrm{~s}^{-1}$ \\
\hline 4 & $\bar{p} p \rightarrow \bar{\Lambda} \Sigma^{0}$ & $\approx 40$ & $\approx 30$ & $\Sigma^{0} \rightarrow \Lambda \gamma$ & $30 \mathrm{~s}^{-1}$ \\
\hline 4 & $\bar{p} p \rightarrow \bar{\Xi}^{+} \Xi^{-}$ & $\approx 2$ & $\approx 20$ & $\Xi^{-} \rightarrow \Lambda \pi^{-}$ & $1.5 \mathrm{~s}^{-1}$ \\
\hline 12 & $\bar{p} p \rightarrow \bar{\Omega}^{+} \Omega^{-}$ & $\approx 0.002^{*}$ & $\approx 30$ & $\Omega \rightarrow \Lambda K^{-}$ & $\approx 4 h^{-1}$ \\
\hline 12 & $\bar{p} p \rightarrow \bar{\Lambda}_{c}^{-} \Lambda_{c}^{+}$ & $\approx 0.1^{*}$ & $\approx 35$ & $\Lambda_{c} \rightarrow \Lambda \pi^{+}$ & $\approx 2 d^{-1}$ \\
\hline
\end{tabular}

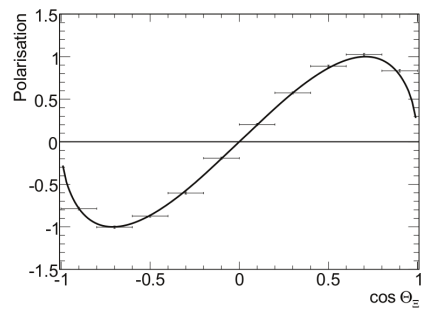

(a)

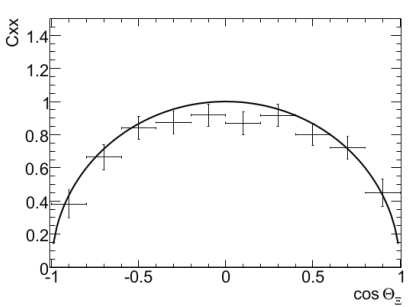

(b)

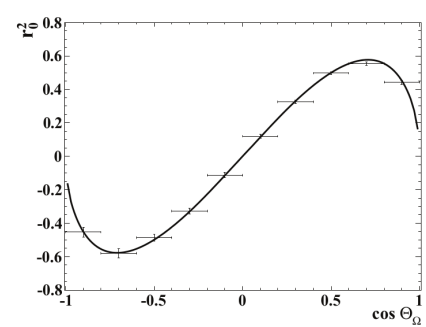

(c)

Figure 2. (a) The polarisation of the $\Xi^{-}$as a function of $\cos \theta_{\Xi}$. (b) The spin correlation $C_{x x}$ in the $x$ direction ( $\hat{m}$ in Fig. 1) of the $\bar{\Xi}^{+}$and the $\Xi^{-}$. (c) The polarisation parameter $r_{0}^{2}$ of the $\Omega$ as a function of $\cos \theta_{\Omega}$. The lines represent the input sine functions (see text) and the points and bars the reconstructed values for 10000 simulated events.

from 1.5 to $15 \mathrm{GeV} / c$, interacting with an internal hydrogen target. The PANDA experiment will have nearly full angular coverage with precise tracking and vertex reconstruction, particle identification, calorimetry, and will benefit from the high luminosity of HESR [11]. Simulation studies with a simplified Monte Carlo framework [12] show excellent prospects with high signal and low background rates and a good detection efficiency across the full phase space for all strange and single-charmed hyperon channels $[8,13]$. The estimated signal rates in Table 1 are calculated using the day- 1 luminosity mode of HESR of $1 \cdot 10^{31} \mathrm{~cm}^{-2} \mathrm{~s}^{-1}$. The only well known cross section is the one for the $\bar{\Lambda} \Lambda$ channel at $1.64 \mathrm{GeV} / c$, whereas the cross sections for the other strange channels are extrapolated from data at other energies. For the heavier hyperons, i.e. $\Omega$ and $\Lambda_{c}$, where no data exist from $\bar{p} p$ annihilation, the values are taken from theoretical predictions which can differ by an order of magnitude $[14,15]$.

Simulation studies of spin observables for $\Lambda, \Sigma, \Xi, \Omega$, and $\Lambda_{c}$ hyperons have been performed as well. Since there exist no data on multi-strange or charmed hyperon channels, a parametrisation based on sine functions was introduced at the event generation stage. These fulfil the requirement of the definition of the production plane (see figure 1) of being equal to zero at $\cos \theta_{Y}= \pm 1$. Figure 2 shows some examples of spin observables of $\Xi$ and $\Omega$, parametrised by $P_{y, \Xi}=\sin 2 \theta_{\Xi}, C_{x x, \Xi}=\sin \theta_{\Xi}$ and $r_{0, \Omega}^{2}=\sin 2 \theta_{\Omega} / \sqrt{3}$. It is clear that these can be accurately reconstructed with PANDA.

The weak decay of the hyperons goes along with comparatively long lifetimes. Their decay vertices can easily be displaced by several tens of centimetres from the production point, setting high demands on precise track reconstruction. The PANDA experiment will include a variety of subdetectors designed for charged track reconstruction, including a Micro Vertex Detector (MVD), a Straw Tube Tracker (STT), and a Gas Electron Multiplier (GEM). The reconstruction can also be supported by a Scintillator Tile Hodoscope (SciTil). Multiple algorithms for pattern recognition and tracking are currently under development with different algorithms for different detectors or detector groups. A 
special focus is given here on the STT, which is a large barrel of 4636 straw tubes. Since its main purpose is to gather transverse information from the tracks, most of these are aligned parallel to the beam axis, while a few layers of straws are skewed by $\pm 2.9^{\circ}$, allowing to gain longitudinal information as well. Simulation studies show that tracks may completely miss one or more of the aforementioned subdetectors, creating only hits within the STT. In these cases, the skewed straws of the STT are the only viable source for a longitudinal momentum information of the track. A tool to support a standalone track finder for the STT is under development which matches the isochrones from the skewed straws with axial information gained from the parallel straws to gather $p_{z}$ information [16].

Using the high luminosity mode of HESR, the mean time between events can be as small as 100 ns while the drift times in the STT can be up to $250 \mathrm{~ns}$, usually causing hits from multiple events to overlap. Events are reconstructed on a software level, using time gaps between hits in one or more of the faster subdetectors, e.g. the MVD. This procedure also yields a first approximation of the start time of each event, $T_{0}$, which in turn is required to determine the isochrones from the straw tube signals. With this first approximation, preliminary tracks will be propagated to detectors with excellent time resolution, e.g. the SciTil, in order to acquire a precise $T_{0}$.

\section{References}

[1] Ellis R K, Stirling W J and Webber B R, QCD and Collider Physics (Cambridge Monographs on particle physics, Nuclear Physics and Cosmology, Cambridge, 1996)

[2] Kohno M and Weise W, Phys. Lett. B 179, 15 (1986); Rubinstein H R and Snellman H, Phys. Lett. B 165, 187 (1985); Furui S and Faessler A, Nucl. Phys. A 486, 669 (1987); Burkardt M and Dillig M, Phys. Rev. C 37, 1362 (1988); Alberg M A et al., Z. Phys. A 331, 207 (1988)

[3] Tabakin F and Eisenstein R A, Phys. Rev. C 31, 1857 (1985); Kohno M and Weise W, Phys. Lett. B 179, 15 (1986); La France P et al., Phys. Lett. B 214, 317 (1988); Timmermanns R G E et al., Phys. Rev. D 45, 2288 (1992); Haidenbauer J et al., Phys. Rev. C 46, 2516 (1992)

[4] Ortega P G et al., Phys. Lett. B 696, 352 (2011)

[5] Doncel M G et al., Nucl. Phys B 38, 477 (1972); Doncel M G et al., Phys. Rev. D 7, 815 (1973)

[6] Yao W M et al., J. Phys. G 33, 1 (2006)

[7] Koch W ed. Nikolic M, Analysis of scattering and decay (Gordon and Breach, New York-LondonParis, 1968)

[8] Thomé E, Multi-Strange and Charmed Antihyperon-Hyperon Physics for PANDA (Ph. D. Thesis, Uppsala Univeryity, 2012)

[9] Johansson T, AIP Conf. Proc. 8th Int. Conf. on Low Energy Antiproton Physics, 95 (2003)

[10] Klempt E et al., Phys. Rep. 368, 119 (2002)

[11] The PANDA collaboration, Technical Performance Report, (2005) https://panda.gsi.de/oldwww/ archive/public/panda_tpr.pdf

[12] The PANDA collaboration, Physics Performance Report, 43 (2009) https://panda.gsi.de/ oldwww/archive/public/panda_pb.pdf

[13] Grape S, Studies on PWO Crystals and Simulations of the $\bar{p} p \rightarrow \bar{\Lambda} \Lambda, \bar{\Lambda} \Sigma^{0}$ Reactions for the PANDA experiment (Ph. D. Thesis, Uppsala University, 2009)

[14] Kaidalov A B and Volkovitsky P E, Z. Phys. C 63, 51 (1994)

[15] Titov A I and Kämpfer B 2008, Phys. Rev. C 78, 025201 (2008); Gornitschnig A T et al., Eur. Phys. J A 42, 43 (2009); He J et al., Phys. Rev. D 84, 114010 (2011); Khodjamirian A et al., Eur. Phys. J A 48, 31 (2012)

[16] Ikegami Andersson W K, private communication 